\title{
EL CASO UBER: ¿SON SUS CONDUCTORES TRABAJADORES AMPARADOS POR LA LEGISLACIÓN LABORAL? COMENTARIO A LA SENTENCIA DE LA CORTE SUPREMA DEL REINO UNIDO DE 19 DE FEBRERO DE 2021, ASUNTO UBER BV AND OTHERS V. ASLAM AND OTHERS [2021] UKSC 5*
}

\author{
Diego Álvarez Alonso \\ Profesor Titular de Derecho del Trabajo \\ y de la Seguridad Social \\ Universidad de Oviedo \\ diegoalonso@uniovi.es
}

De un tiempo a esta parte viene estando de actualidad discutir desde las perspectivas jurídica, periodística y hasta política si las personas que prestan servicios «a demanda» vinculadas a plataformas digitales de reparto o transporte de viajeros, como Glovo, Deliveroo, Uber, Lyft o Cabify, son trabajadores asalariados de dichas plataformas sometidos a la legislación laboral y de Seguridad Social referidas a las relaciones de trabajo por cuenta ajena, o son, como aquellas empresas sostienen, trabajadores autónomos al margen de la aplicación de tales normas. Tras una primera fase de controversia plenamente abierta y algunas resoluciones judiciales dispares sobre el particular, parece que las respuestas empiezan a decantarse a través de iniciativas de intervención legislativa todavía en construcción y, principalmente, de sentencias que comienzan a establecer jurisprudencia en la materia desde la cúspide del sistema jurisdiccional.

En nuestro país, la Sentencia del Tribunal Supremo 805/2020, de 25 de septiembre (r. 4746/2019), dictada en un asunto relativo a un repartidor ligado a la plataforma Glovo, ha unificado doctrina considerando que se trata de un trabajador asalariado y, por tanto, de una relación laboral

* El presente trabajo se ha realizado en el marco de la participación del autor en el proyecto de investigación «La jurisprudencia del Tribunal Supremo en materia laboral y social: ámbito funcional, trayectoria y aportaciones más significativas» (Ref.: DER-2016-80327-P). 
incluida dentro del ámbito de aplicación del Derecho de Trabajo, rechazando la calificación como trabajador autónomo económicamente dependiente pretendida por la empresa. Paralelamente, en otros ordenamientos de nuestro entorno se han dictado recientemente sentencias relevantes referidas al caso - algo distinto, pero próximo- de la conocida plataforma de transporte urbano de viajeros Uber. Así, en Francia, la Corte de Casación se ha pronunciado a favor del estatus laboral de los conductores adscritos a dicha plataforma [Arrêt n 374, 4/03/2020 (19-13.316), Chambre Sociale (arrêt «Uber»)], y, en el Reino Unido, se ha confirmado la aplicación de normas laborales a esta forma de prestación de servicios en la Sentencia de la Corte Suprema de 19 de febrero de 2021, que es de la que aquí se da cuenta específicamente [Uber BV and others $v$. Aslam and others (2021) UKSC 5; Appeal [2018] EWCA Civ 2748].

Esta sentencia de la Corte Suprema británica confirma el sentido de la decisión adoptada en su momento en la primera instancia y confirmada posteriormente por el Employment Appeal Tribunal y la Court of Appeal declarando que los conductores de Uber personados en el proceso son trabajadores de la empresa propietaria de la plataforma y no trabajadores plenamente independientes como esta sostenía, siendo, por consiguiente, aplicables normas laborales como las relativas a salario mínimo, tiempo de trabajo y descansos (incluido el derecho a vacaciones anuales retribuidas) y el derecho a no sufrir represalias por la denuncia de actuaciones irregulares («whistleblowing»). Pues bien, el último recurso de las empresas concernidas topa ahora nuevamente con la respuesta adversa de los lores de la Corte Suprema en una decisión que parece dejar zanjada de manera definitiva en Gran Bretaña la cuestión relativa a la calificación de los conductores de Uber como trabajadores (workers) con acceso a los derechos laborales mencionados.

En todo caso, conviene precisar que estos trabajadores se clasifican por el momento en la categoría de workers, que no es plenamente equivalente a la del trabajador asalariado por cuenta ajena utilizada en el ordenamiento español y la mayoría de los sistemas europeos, ni tiene exactamente los mismos efectos. En el Derecho británico, hay una primera distinción entre los profesionales autónomos que trabajan individualmente por cuenta propia (independent self-employed), que quedan totalmente al margen del radio de acción del Derecho del Trabajo, y, por otro lado, los trabajadores (workers), categoría que engloba, a su vez, a la del trabajador asalariado subordinado y por cuenta ajena en sentido estricto (employee), pero también a aquellas otras personas que presten personalmente servicios para 
una contraparte de un modo que no pueda calificarse como libre ejercicio profesional o actividad empresarial plenamente autónoma y por cuenta propia [Employment Rights Act 1996, Section 230(3)]. Para toda esta «macro-categoría» de workers hay un mínimo común de derechos y normas laborales (floor of rights) igualmente aplicables a todos ellos, con independencia de que se trate o no de trabajadores asalariados subordinados y con contrato de trabajo, y que son los ya referidos a salario mínimo, tiempo de trabajo, descansos y protección frente a discriminación y represalias (Employment Rights Act 1996, National Minimum Wage Act 1998 y Working Time Regulations 1998). Ahora bien, a partir de ahí hay que distinguir: por un lado, los employees con contrato de trabajo y que responden a las notas del trabajo subordinado por cuenta ajena tendrían una genuina relación laboral plenamente sometida a la legislación laboral en su integridad, mientras que, por otro, a los restantes workers que no alcanzan el estatus de employee se les aplicarían selectivamente solo aquellos derechos y normas laborales que conforman el estatuto básico común.

Dicho de modo más gráfico, aunque quizá menos preciso, en el Derecho británico existen como categorías contrapuestas la de los empleados subordinados por cuenta ajena (employees) con relación laboral a la que resulta aplicable el Derecho del Trabajo en su totalidad, y, de otra parte, la de los trabajadores independientes por cuenta propia, completamente excluidos del ámbito de aplicación de aquel. Ahora bien, como la propia sentencia comentada subraya, entre esas dos categorías opuestas emerge una tercera «clase de trabajadores autoempleados, pero que prestan servicios como parte de una actividad profesional o empresarial regida por persona o entidad distinta». En este contexto, aunque «algunos derechos, como la protección frente al despido injustificado, se limitan a quienes son empleados bajo un contrato de trabajo, otros se aplican a todos los workers», incluidos los situados en zona de frontera entre el trabajo asalariado y el verdaderamente independiente.

Es dentro de este radio precisamente donde la sentencia comentada sitúa a los conductores de vehículos de la plataforma Uber al calificarlos como workers, quedándoles así garantizado el acceso a los derechos antes aludidos en materia salarial, de tiempo de trabajo y protección frente a represalias, que es lo que aquellos reclamaban concretamente. No obstante, también conviene aclarar que, si bien la sentencia no califica a estos conductores como trabajadores subordinados y por cuenta ajena en sentido estricto (employees), tampoco excluye ni impide que ello pueda suceder más adelante. Es más, la propia sentencia comentada parece dar pie 
en algún pasaje a considerar que a estos trabajadores habría que calificarlos no solo como workers, sino probablemente también como verdaderos trabajadores asalariados o employees, y la fundamentación que en ella se contiene ofrece numerosos argumentos en tal sentido, como se verá a continuación.

La sentencia de la Corte Suprema parte de una detallada y significativa descripción del «sistema Uber», del funcionamiento de la aplicación, de la forma de organización y prestación de los servicios de transporte, del procedimiento de cálculo de las tarifas para el usuario y de las ganancias correspondientes a los conductores y a la empresa titular de la plataforma, de la gestión de los pertinentes cobros y pagos, de las condiciones en que los conductores se vinculan a la plataforma, de los acuerdos y documentos contractuales suscritos entre las distintas partes implicadas, e incluso del modo concreto en que se ejecuta el trabajo de transporte de pasajeros, se elige la ruta a seguir y se resuelven eventuales incidencias. Por otro lado, recordando jurisprudencia anterior al respecto, se señala que en el ordenamiento británico (como en el español) la delimitación legal de la figura del trabajador y del campo de aplicación de las normas laborales es imperativa, e indisponible para la autonomía privada, por interés o decisión de las partes de la relación contractual, prevaleciendo a estos efectos siempre la calificación que corresponda a la realidad de las cosas, por encima incluso del nomen iuris o la apariencia jurídica formal de los contratos suscritos.

Con esos mimbres, la sentencia pasa en efecto a analizar las circunstancias fácticas para determinar si son subsumibles dentro de la definición legal de trabajador (worker). En este sentido, se identifican como notas definitorias clave las de subordinación y dependencia del trabajador con respecto a otra persona o entidad en relación con el trabajo realizado. Además, se identifica como «piedra de toque de la subordinación y dependencia» el grado de control ejercido por el «empleador putativo» sobre el trabajo realizado por la persona concernida, de suerte que, cuanto más intenso sea ese control, más fuerte será la razón para clasificarla dentro de la categoría legal de trabajador (worker). Se cita a estos efectos, por cierto, alguna sentencia del Tribunal de Justicia de la Unión Europea sobre el concepto comunitario de trabajador, pese a haberse consumado ya el Brexit en la fecha en que la Corte Suprema del Reino Unido dicta la resolución comentada.

Pues bien, la Corte Suprema reconoce que los conductores de Uber cuentan con un grado sustancial de autonomía o independencia en lo que 
se refiere al volumen, tiempo y lugar de trabajo, contando con gran flexibilidad para decidir cuánto, cuándo y dónde trabajar. En este sentido, se admite que el carácter extremadamente irregular, discontinuo o meramente esporádico de la prestación de servicios para otros pudiera, según las circunstancias del caso, determinar la exclusión de la aplicación de las normas laborales. Ahora bien, no siempre es así, pues, pese a concurrir este tipo de circunstancias, se viene dando encaje en el concepto de trabajador (worker o incluso employee) a supuestos tales como los de los trabajadores eventuales (casual workers) y los trabajadores de temporada (seasonal workers), a los que se vienen a asimilar ahora en cierto modo los trabajadores «a demanda» vinculados a plataformas digitales.

Ello es así porque, dejando al margen la referida flexibilidad horaria, en lo demás se aprecia acertadamente que los conductores de Uber realizan su trabajo con un altísimo grado de subordinación y dependencia, funcional y económica, con respecto a la empresa titular de la plataforma. Adicionalmente, la sentencia resalta la importancia de aquilatar la posición de las partes implicadas en lo relativo a cuestiones como la fijación de los precios y la responsabilidad por el servicio ante los pasajeros, valorando en particular hasta qué punto los conductores tienen la posibilidad de ofrecer sus servicios al cliente por sí mismos, por cuenta propia y como empresarios independientes. Se trata de consideraciones que, si bien se mira, entroncan con lo que en el Derecho del Trabajo español denominamos ajenidad, y, en especial, con la explicación de esta nota definitoria del trabajo asalariado desde la perspectiva de la «ajenidad en el mercado».

$\mathrm{Al}$ respecto, son varios los indicios e indicadores que trae a colación la Corte Suprema. Como primero y más importante de entre todos estos elementos de juicio se subraya que es la empresa Uber la que fija unilateralmente las tarifas cobradas a los pasajeros, las compensaciones abonadas a los conductores por trayecto realizado y la comisión retenida por la entidad titular de la plataforma por cada servicio completado. Ello se hace de manera homogénea, según los designios de la empresa Uber, con un sistema aplicado de manera estandarizada a todos los conductores, sin margen alguno de intervención o negociación individualizada por parte de estos en la determinación de los precios con que sus servicios se ofrecen al mercado. Se trata de lo que en un sector de la doctrina laboralista española hemos comenzado a denominar ajenidad en la fijación de los precios y tarifas, circunstancia que apunta al estatus laboral de la relación, ya que, si el trabajador prestador del servicio no tiene ninguna capacidad propia y autónoma de decisión en la fijación del precio cobrado al cliente, carecien- 
do de poder alguno en la determinación de los márgenes comerciales de su actividad, difícilmente se le podrá considerar como emprendedor por su cuenta. Se trata de un indicador que ha tenido en cuenta asimismo el Tribunal Supremo español en la Sentencia 805/2020 referida al asunto Glovo, destacando que, también en este caso, es la titular de la plataforma quien establece, de manera unilateral, predeterminada y homogéneamente estandarizada para todos los prestadores del servicio, no solo el precio al consumidor final, sino también la remuneración a los repartidores —igualmente estandarizada y sin margen de variación negociable-, así como los términos económicos de los acuerdos con las empresas proveedoras asociadas. Dada esta absoluta falta de poder para decidir por sí mismos en lo relativo a las propias ganancias y al precio de lo que se oferta en el mercado, resulta prácticamente ridículo pretender que se considere a estos trabajadores como verdaderos empresarios independientes.

En segundo lugar, en la misma línea, la sentencia subraya que los términos contractuales con arreglo a los que los conductores desarrollan sus servicios se dictan unilateralmente por Uber. No solo se requiere a los prestadores del servicio la aceptación íntegra de los formularios estandarizados de acuerdos por escrito, sino que los términos en que ha de desenvolverse el transporte de pasajeros son igualmente impuestos por Uber, sin que los conductores puedan decir o modificar nada al respecto. Así, la evidencia de que no hay negociación bilateral entre empresarios colaboradores independientes, sino, más bien, fijación unilateral de contratos de adhesión y condiciones de prestación del servicio que se imponen en masa e imperativamente por decisión de la plataforma, apunta, al menos indiciariamente, a la existencia de la ajenidad y subordinación propias de la relación laboral. Concurrentes en el mismo sentido son las apreciaciones que se hacen en relación con el control de la información sobre los pasajeros y los trayectos solicitados mediante la aplicación, que es ejercido de modo absoluto por Uber, restringiéndose implícita y explícitamente la comunicación directa entre el conductor y el cliente final, que solo puede hacerse a través de dicha aplicación y dentro de las condiciones de tiempo y modo fijadas por esta. Esta ajenidad en la información operaría también como indicador significativo de dependencia de los conductores con respecto a la empresa propietaria de la plataforma.

Ese control de las relaciones con la clientela exclusivamente por la empresa Uber, quedando vedada toda interacción comercial directa del conductor con los pasajeros, es reflejo de lo que la doctrina laboralista española denomina ajenidad en el mercado, y se aprecia sobremanera en el 
modo en que se articulan el cobro de las tarifas, la facturación, el pago por los servicios prestados y la gestión de quejas. Así, por ejemplo, estas últimas han de vehicularse siempre a través de la aplicación, siendo la empresa Uber la que responde en todo caso, nunca el conductor, procediendo la empresa, cuando lo estima oportuno, a la devolución de importes por servicios deficientes, y normalmente sin repercutirlo luego sobre los chóferes, lo que es muestra clara de la ajenidad en los riesgos. El único contacto autorizado entre conductor y pasajero tras la conclusión del servicio es a efectos de recuperación de objetos olvidados en el vehículo.

Por otra parte, la sentencia reconoce que, a primera vista, pudiera parecer que los conductores cuentan con una amplia flexibilidad para decidir cuánto y cuándo quieren trabajar, sin estar sometidos a horario fijo, y con ciertas posibilidades de rechazar encargos de trabajo, desconectándose temporalmente de la aplicación o no aceptando las solicitudes de recogida de pasajero remitidas por aquella. Sin embargo, esa libertad es más aparente que real, porque Uber ostenta en último término el control por diversas vías. De entrada, retiene la información sobre el trayecto solicitado, de manera que el conductor carece de elementos de juicio para sopesar si dicho itinerario le interesa o no. Pero, sobre todo, Uber monitoriza a través del software de la plataforma las tasas de aceptación y rechazo de trayectos por parte de los conductores, procesando de manera automatizada las métricas resultantes, que, cuando son negativas, se traducen en mensajes de advertencia o amonestación enviados al trabajador que no rinde lo suficiente, o incluso penalizaciones que parecen auténticas sanciones disciplinarias, en la forma de una «desactivación temporal» en la que aquel queda privado de encargos entrantes y, por tanto, de las consiguientes ganancias (inicialmente, por un periodo de diez minutos), o hasta comportando la desconexión y definitiva expulsión de la plataforma, lo que vendría a ser equivalente a un despido, a fin de cuentas. Alegaba la empresa que ello se justifica en aras a la garantía de la calidad del servicio, a lo que la sentencia responde que la cuestión no es esa, sino si tal sistema de control coloca a los conductores en una posición de subordinación a Uber. A lo que el Tribunal contesta con contundencia: «It pleinly does»; es decir, sí, «plenamente».

Esa apreciación sobre la palmaria existencia de subordinación se refuerza con alusión a otros elementos. Así, se enfatiza, por ejemplo, que Uber determina el tipo de coches que pueden ser utilizados para el servicio (clase, modelos, condiciones y hasta el color) o que proporciona instrucciones precisas sobre cómo debe ser el trato correcto y educado hacia el 
cliente. Pero también, sobre todo, que la propia aplicación dirige el modo de ejecución de la prestación del trabajo. En este sentido, la aplicación indica al conductor el trayecto concreto que ha de seguir entre el punto de recogida del pasajero y el destino, sin poder desviarse o elegir una ruta alternativa, aspecto que es sometido a vigilancia por la empresa mediante el seguimiento GPS. A ello se une la existencia de un sistema de valoración o puntuación de los conductores (ratings) por parte de los clientes a través de la misma aplicación. Las métricas resultantes son procesadas por el software de la plataforma, junto a las antes citadas sobre aceptación y cancelación de encargos, de modo que los conductores con bajas puntuaciones reciben advertencias o amonestaciones, y, en último término, sanciones más severas, incluida la extinción de la relación y expulsión de la plataforma. A este respecto, puede encontrarse un indudable paralelismo con la decisión del Tribunal Supremo español en su Sentencia 805/2020, relativa al asunto Glovo, donde también se toman como indicios e indicadores de estatus laboral el seguimiento de los repartidores a través de GPS, la existencia de un sistema de evaluación y puntuación de aquellos por la clientela, la monitorización del número de encargos realizados y rechazados, así como de los tiempos de reparto, y, sobre todo, el procesamiento de todos estos datos a través de la plataforma, que, a partir de ellos, organiza la distribución del trabajo y el posicionamiento de los repartidores para recibir nuevos encargos, e incluso adopta penalizaciones contra los repartidores que resultan menos productivos.

Pudiera tal vez objetarse que el seguimiento por GPS o la existencia de un sistema de evaluaciones por parte de la clientela no tienen por qué ser en sí mismos indicios concluyentes de subordinación o característica exclusiva del trabajo asalariado, por cuanto también hay transportistas, repartidores y otros profesionales verdaderamente autónomos que pueden ser objeto de diversos sistemas de valoración sobre sus prestaciones y la calidad del servicio ofrecido en el mercado (mediante las habituales encuestas de satisfacción, rankings elaborados por terceros, etc.). No obstante, como apunta la sentencia comentada, esta línea argumentativa podría ser válida si se trata de las típicas valoraciones, calificaciones y certificaciones que se ponen a disposición de la clientela como información para ayudar a tomar decisiones sobre qué producto o servicio contratar y con quién (por ejemplo, las estrellas de los hoteles, la Q de calidad turística de los establecimientos hosteleros o las puntuaciones de restaurantes en aplicaciones como TripAdvisor). Ahora bien, la sentencia subraya, decisiva y acertadamente, que el uso que Uber hace de las evaluaciones de los 
clientes es materialmente diferente. De hecho, las valoraciones de los conductores no se muestran a la clientela como un modo de orientar sus decisiones, y los pasajeros no pueden elegir entre unos y otros conductores en función de su puntuación. Por el contrario, el destino de las puntuaciones es exclusivamente su procesamiento puramente interno por la plataforma, para tomar las decisiones antes aludidas sobre eventuales penalizaciones a los trabajadores y, más en general, para la dirección y organización colectiva del trabajo a través de los sistemas automatizados incorporados a la aplicación. A juicio de la Corte Suprema, «esta es una clásica forma de subordinación característica de la relación propiamente laboral».

Bien es verdad que esa subordinación se mantiene en términos clásicos en cuanto a su esencia y significación, pero se articula por métodos nuevos que de algún modo podrían despistar al intérprete que permanezca anclado con excesiva estrechez de miras a los indicios clásicos de laboralidad, como la sujeción a horario y lugar de trabajo fijos, la imposibilidad de rechazar encargos o la transmisión directa de órdenes e instrucciones explícitas a través de un organigrama jerárquico de directivos y mandos intermedios. En el mundo de la economía de plataformas digitales, el trabajo se descompone en «microtareas» repartidas entre una multitud de trabajadores que cobran en función de los servicios realizados, lo que garantiza que haya siempre alguno que acepte el servicio que otro pueda dejar pasar (crowdsourcing), y se realiza el ajuste instantáneo «a demanda» de las necesidades de fuerza de trabajo a través de la organización digital de actividad. Todo ello hace irrelevante la sujeción a horario (y lugar) de trabajo fijo, la exclusividad o la libertad de aceptación o rechazo de encargos. Además, lo que se ha dado en llamar la organización automatizada o «algorítmica» del trabajo hace innecesaria la existencia de cadena de mando o estructura jerárquica en la empresa, pues el software informático o aplicación, gracias a la inteligencia artificial, puede realizar perfectamente, e incluso más eficazmente, las funciones de programación, dirección y coordinación de la actividad, así como las de vigilancia y control del trabajo, a través de sistemas de puntuación, métricas y asignación automatizada de tareas y penalizaciones como el que se ha descrito antes, y contando, en su caso, con la delegación de la supervisión del servicio en las valoraciones de la propia clientela. Los casos de Uber y Glovo, siguiendo la descripción de las sentencias aquí aludidas, son buenos ejemplos para explicar en qué consiste esa organización algorítmica del trabajo (algorithmic management) e ilustran a la perfección cómo estos sistemas automatizados pueden hacer desaparecer elementos tales como el horario fijo, la estruc- 
tura de mando en la empresa o la vigilancia y control de la actividad laboral por los superiores, esto es, los que venían operando como indicios más tradicionales en torno al estatus asalariado y por cuenta ajena del trabajador. Sin embargo, la ausencia de aquellos viejos indicios no implica por sí misma la desaparición de la subordinación característica del trabajo asalariado. Lo que ocurre es que aquellos elementos clásicos de la organización laboral han sido reemplazados por estos otros métodos más novedosos, a través de los que puede persistir —o incluso intensificarse- la subordinación o dependencia del trabajador, aunque por vías más sutiles y difíciles de apreciar con claridad.

A la vista de todo lo dicho, la Corte Suprema concluye con rotundidad que a los conductores de Uber les corresponde el estatus de workers, que conlleva la aplicación selectiva de ciertos derechos laborales, respondiendo así a lo que concretamente se planteaba en el litigio resuelto. Ahora bien, a la vista de lo comentado, es claro que la sentencia va todavía más allá, pues ofrece indicaciones nada veladas que invitan a considerar que estos trabajadores son además employees, es decir, trabajadores asalariados con relación laboral íntegramente sujeta al Derecho del Trabajo, si bien esto no se incorpora al contenido del fallo, ya que la cuestión no se había planteado en esos términos por los demandantes. Por otro lado, más allá del primer nudo gordiano relativo a la calificación de esta prestación de servicios como autónoma o dependiente, la sentencia se adentra también en un segundo problema espinoso que se da en este y otros supuestos próximos de trabajo ligado a plataformas digitales, relacionado con la intrínseca intermitencia o discontinuidad de estas prestaciones de servicios.

En efecto, en el caso de los conductores de Uber como en el de los repartidores de Deliveroo o Glovo, no se trabaja con la continuidad propia de un horario laboral fijo y con una distribución regular a lo largo de la jornada diaria y semanal. Se trabaja de manera extremadamente flexible, discontinua e irregular, exclusivamente en los periodos en que el trabajador se conecta o se activa dentro de la aplicación, poniéndose entonces a disposición para la recepción de encargos, que pueden entrar - $\mathrm{O}$ no- - con una frecuencia no prevista de antemano. Es más, aun dentro de estos periodos de disponibilidad o activación, el trabajo solo se realiza de manera materialmente efectiva cuando se acepta y comienza a desarrollarse el correspondiente trayecto de recogida y transporte de pasajeros o de productos repartidos a domicilio. Así, entre la ejecución de un encargo y la entrada del siguiente suele mediar un tiempo de espera, en el que el trabajador puede permanecer conectado a la aplicación, pero inactivo o 
dedicado a otros menesteres, e incluso quizá estar trabajando en otra cosa, o hasta para empresas de la competencia. Con este contexto, se plantearon en el litigio aquí comentado dos cuestiones: de una parte, ¿cuándo se entiende iniciado y por qué periodos se considera activo y subsistente el contrato entre las partes sobre el que se aplica la legislación laboral con las consiguientes responsabilidades para la empresa?; de otra, ¿qué es lo que hay que considerar como tiempo de trabajo efectivo a efectos de aplicación de la legislación laboral, y especialmente de las normas sobre salario mínimo, y qué consideración merecen las interrupciones y los tiempos de espera en que el trabajador permanece conectado, pero inactivo? Se trata de interrogantes que aún no se han suscitado tan directamente ante los órganos judiciales españoles, pero que a buen seguro se plantearán a no mucho tardar, por lo que resulta interesante ver la respuesta dada por la Corte Suprema británica, en tanto que posible fuente de inspiración o elemento de contraste con la que en el futuro haya de darse en el ordenamiento español, ya sea por el legislador o por jueces y tribunales.

Sobre el primero de esos interrogantes, la Corte concluye que un conductor puede ser considerado como trabajador (worker) de la empresa Uber desde el momento en que suscribe con dicha entidad un contrato en que se compromete a la prestación de servicios de conducción para ella. No se estima necesario para la adquisición de ese estatus de trabajador que, adicionalmente, el conductor se haya puesto ya efectivamente a disposición y se encuentre listo para la aceptación de trayectos, como en cambio consideró el employment tribunal. Ahora bien, la Corte Suprema considera «razonable» valorar esta circunstancia, tal y como había hecho el citado tribunal, como una posible condición suplementaria a la hora de determinar si el trabajador se encuentra efectivamente trabajando bajo dicho contrato. A partir de ahí, pasa a analizarse qué periodos de tiempo deben contar como tiempo efectivo de trabajo y cuáles no. Con cita de jurisprudencia del Tribunal de Justicia de la Unión Europea al respecto (STJUE de 21 febrero 2018, Ville de Nivelles c. Matzak, asunto C-518/15), se resuelve que ha de considerarse que no solo es tiempo de trabajo el que media entre la recogida de cada pasajero y la finalización del trayecto correspondiente, sino que también lo son los lapsos de espera en que el conductor permanece conectado a la aplicación y disponible para recibir nuevas solicitudes de recogida y transporte de viajeros. Así pues, estos periodos temporales de disponibilidad en interés de la empresa, aunque sin desempeño laboral efectivo, deben computarse igualmente como tiempo de trabajo efectivo, particularmente a efectos de la aplicación de la normativa sobre salario mínimo. 
No obstante, la empresa Uber se oponía radicalmente a esta interpretación con una alegación de cierta entidad. Se argumentaba que difícilmente puede considerarse que esos tiempos de espera y disponibilidad son tiempo de trabajo efectivo para la empresa Uber cuando en ellos el conductor puede permanecer simultáneamente conectado a otras aplicaciones distintas de las empresas competidoras que operan servicios similares (Lyft, Cabify), y para las que hipotéticamente el trabajador podría estar trabajando en paralelo. Pues bien, la Corte Suprema parece aceptar que, de ser ese verdaderamente el caso, cabría excepcionar lo dicho anteriormente, y no sería posible tener en cuenta esos tiempos de disponibilidad como tiempo de trabajo efectivo al servicio y bajo la responsabilidad únicamente de Uber. Ahora bien, se señala que este es un planteamiento por el momento meramente hipotético, ya que, en la práctica, Uber venía monopolizando esta actividad de servicios de transporte urbano de viajeros solicitados mediante aplicación en la ciudad de Londres, sin hueco en el mercado para otros competidores, de manera que sus conductores venían trabajando en exclusiva para aquella empresa, con un volumen de encargos y dedicación que venía a hacer puramente quimérica la supuesta (e irreal) posibilidad de ponerse a disposición de otras plataformas. Queda así soslayada esta objeción en el caso concreto enjuiciado, pero se deja abierta y sin resolver la intrincada situación de trabajo simultáneo para varias plataformas, que puede ser meramente hipotética en la ciudad de Londres, pero que podría ser una realidad tal vez tangible en otros lugares, como, sin ir más lejos, Madrid, donde este mercado se encuentra repartido entre, al menos, dos empresas competidoras (Uber y Cabify).

En suma, la comentada Sentencia de la Corte Suprema del Reino Unido de 19 de febrero de 2021 merece ser destacada como uno de los primeros hitos de la jurisprudencia comparada en materia de trabajo vinculado a plataformas digitales, junto a - entre otras- la Sentencia de nuestro Tribunal Supremo 805/2020, de 25 de septiembre. Ambas se adscriben a la línea interpretativa proclive a recalificar a esta clase de prestadores de servicios «a demanda» (conductores y repartidores), pretendidamente autónomos y carentes de amparo legal, como trabajadores con derechos, protegidos —en mayor o menor grado- por la imperativa aplicación de las normas laborales. En España, la sentencia citada concreta lo anterior, por el momento, en el caso de los repartidores de Glovo, pero la lectura de aquella al lado de la comentada resolución británica podría permitir entrever una solución por los mismos derroteros para el también paradigmático caso de Uber o incluso para otros igualmente próximos. Porque, pese 
a la diversa idiosincrasia jurídica, ambas resoluciones manejan argumentaciones similares al respecto que pueden verse como bien encaminadas y que resultan concurrentes, aun partiendo de contextos socioeconómicos y culturales muy distintos. Entre otras, las que señalan que la no concurrencia de algunos indicios clásicos de la subordinación y ajenidad propias del trabajo asalariado (horario y lugar de trabajo fijo, integración en la jerarquía empresarial, imposibilidad de rechazo de encargos) no implica que aquellas notas hayan dejado de estar presentes a través de medios más sofisticados y sutiles de organización algorítmica y apropiación empresarial de la fuerza de trabajo, que incluso pueden redoblar la intensidad de la dependencia y alienación del trabajador, aunque de una forma distinta de lo acostumbrado. De otra parte, la sentencia comentada ofrece también algunas ideas y perspectivas de análisis diferentes de las manejadas por la doctrina judicial española y con las que vale la pena hacer el cotejo para ensanchar la mirada.

Finalmente, si en nuestro país los órganos jurisdiccionales (y, aparentemente, las iniciativas legislativas que se anuncian como inminentes) se han centrado hasta el momento solo en el nudo gordiano inicial de la calificación jurídica del trabajo en plataformas digitales como relación laboral, la sentencia del Reino Unido avanza ya que, más allá de ello, hay una mucho más amplia gama de cuestiones problemáticas que se suscitan en este terreno, como las relativas a los tiempos de espera entre encargos y la determinación de los tiempos computables como de trabajo efectivo, la irregularidad intrínseca de la prestación de servicios o las renovadas posibilidades de trabajo simultáneo para varias empresas distintas. A las que cabría agregar cuestiones como, por ejemplo, la garantía de una carga de trabajo y unos ingresos mínimos, la difícil articulación de la protección de la seguridad y salud en el trabajo o la constitución de la representación de los trabajadores en contextos sin un centro de trabajo físico como referencia, entre otras. En definitiva, la sentencia comentada y las demás coetáneas aquí aludidas son indudablemente leading cases muy relevantes, si bien estamos ante una temática en la que cabe esperar ulteriores hitos de similar o incluso mayor envergadura. 